\title{
A characterization of finite soluble groups by laws in two variables*
}

\author{
John N. Bray, John S. Wilson and Robert A. Wilson
}

\begin{abstract}
Define a sequence $\left(s_{n}\right)$ of two-variable words in variables $x, y$ as follows: $s_{0}(x, y)=$ $x, s_{n+1}(x, y)=\left[s_{n}(x, y)^{-y}, s_{n}(x, y)\right]$ for $n \geqslant 0$. It is shown that a finite group $G$ is soluble if and only if $s_{n}$ is a law of $G$ for all but finitely many values of $n$.
\end{abstract}

\section{Introduction}

It is well known that a finite group $G$ is nilpotent if and only if $e_{n}$ is a law in $G$ for all sufficiently large integers $n$, where the words $e_{n}$ in the free group on $x, y$ are defined inductively by

$$
e_{0}(x, y)=x, \quad e_{n+1}=\left[e_{n}(x, y), y\right] \text { for } n \geqslant 0
$$

(see for example [?, 12.3 .4 on p. 358]). In [?] a sequence $v_{n}$ of words in two variables was given with the property that a finite group $G$ is soluble if and only if $v_{n}$ is a law in $G$ for all sufficiently large integers $n$. However the sequence $\left(v_{n}\right)$ does not have a simple recursive definition like that of the sequence $\left(e_{n}\right)$ : it depends on an enumeration of the elements of a subgroup of the free group, and there is no easily described relationship between its consecutive terms. It was shown in [?] that this deficiency can be partly remedied at the cost of allowing four variables instead of two. Further progress on sequences of laws in two variables which characterize finite soluble groups was made in [?] and the preprint [?]. Here we prove the following result.

Theorem A Define the sequence $s_{n}$ of laws in two variables $x, y$ by

$$
s_{0}=x, \quad s_{n+1}(x, y)=\left[s_{n}(x, y)^{-y}, s_{n}(x, y)\right] \text { for all } n \geqslant 0 .
$$

Then a finite group $G$ is soluble if and only if $s_{n}$ is a law in $G$ for all sufficiently large integers $n$.

*Mathematics Subject Classification 20D10, 20D06 
Our notation for conjugates and commutators is as follows: $x^{y}=y^{-1} x y, x^{-y}=$ $y^{-1} x^{-1} y,[x, y]=x^{-1} x^{y}$.

In [?], a somewhat more complicated recursively defined sequence is given that characterizes finite soluble groups: its properties are established as a consequence of Thompson's classification [?] of the minimal simple groups, together with a fixed-point theorem whose proof depends on deep results in algebraic geometry and a substantial amount of computation. Our proof depends on the classification of the minimal simple groups, but it is otherwise self-contained.

Since the values of $s_{n}$ in a group $G$ evidently lie in the $n$th term of the derived series for $G$, soluble groups of derived length $n_{0}$ satisfy the law $s_{n}$ for all $n \geqslant n_{0}$. To prove the converse, one considers a minimal counter-example: such a group is a minimal simple group. For each element $u$ of a group $G$ define the map $\theta_{u}: G \rightarrow G$ by $\theta_{u}(w)=\left[w^{-u}, w\right]$. Thus the images of an element $w$ of $G$ under the powers of the map $\theta_{u}$ are just the terms of the sequence $\left(s_{n}(w, u)\right)_{n \geqslant 1}$. Hence Theorem $\mathrm{A}$ is an immediate consequence of the following result.

Theorem B Every minimal simple group $S$ has an involution $u$ and a non-empty subset $X$ with $\theta_{u}(X) \subseteq X$ and $1 \notin X$.

Thompson's classification of the minimal simple groups implies that the minimal simple groups are to be found among the groups $\mathrm{L}_{2}(q)$ with $q$ a prime power such that $q \geqslant 5$, the Suzuki groups $\mathrm{Sz}(q)$ with $q=2^{2 m+1} \geqslant 8$, together with the group $\mathrm{L}_{3}(3)$. Sections 2 and 3 are dedicated to groups $\mathrm{SL}_{2}(F)$ and Suzuki groups respectively: they contain results giving immediately the assertion of Theorem $\mathrm{B}$ for groups of types $\mathrm{L}_{2}(q)$ and $\mathrm{Sz}(q)$. The case of $\mathrm{L}_{3}(3) \cong \mathrm{SL}_{3}(3)$ is easy and we deal with it here. Let $u, w$ be the matrices in $\mathrm{SL}_{3}(3)$ given below:

$$
u=\left(\begin{array}{ccc}
1 & 0 & 0 \\
0 & 2 & 0 \\
0 & 0 & 2
\end{array}\right) \quad \text { and } \quad w=\left(\begin{array}{ccc}
1 & 1 & 0 \\
1 & 0 & 1 \\
0 & 1 & 1
\end{array}\right)
$$

Clearly $u^{2}=1$ and it is routine to verify that $\theta_{u}^{4}(w)=w \neq \theta_{u}^{2}(w)$; thus the set $X=$ $\left\{w, \theta_{u}(w), \theta_{u}^{2}(w), \theta_{u}^{3}(w)\right\}$ is permuted as a 4-cycle by $\theta_{u}$ and certainly does not contain the identity matrix.

\section{Groups of type $\mathrm{SL}_{2}(F)$}

In order to prove Theorem B for groups $S \cong \mathrm{L}_{2}(q)$ it is sufficient to work with groups $\mathrm{SL}_{2}(F)$ with $F$ finite of order at least 5 and to find an element $u \in \mathrm{SL}_{2}(F)$ and a subset 
$X$ of $\mathrm{SL}_{2}(F)$ with $u^{2}= \pm 1, \theta_{u}(X) \subseteq X$ and $\pm 1 \notin X$. Let $F$ be an arbitrary field and fix the element

$$
u=\left(\begin{array}{cc}
0 & 1 \\
-1 & 0
\end{array}\right)
$$

of $\mathrm{SL}_{2}(F)$. Thus $u^{2}=-1$. We recall that $\theta_{u}: \mathrm{SL}_{2}(F) \rightarrow \mathrm{SL}_{2}(F)$ is the map defined by $\theta_{u}(w)=\left[w^{-u}, w\right]$. Let

$$
w=\left(\begin{array}{ll}
a & b \\
c & d
\end{array}\right) \text { and } \theta_{u}(w)=\left(\begin{array}{cc}
A & B \\
C & D
\end{array}\right)
$$

Easy calculations show that

$$
\left[u, w^{-1}\right]=\left(\begin{array}{cc}
c^{2}+d^{2} & -a c-b d \\
-a c-b d & a^{2}+b^{2}
\end{array}\right), \quad[u, w]=\left(\begin{array}{cc}
a^{2}+c^{2} & a b+c d \\
a b+c d & b^{2}+d^{2}
\end{array}\right)
$$

and so since $\theta_{u}(w)=\left[u, w^{-1}\right][u, w]$ we have

$$
\begin{aligned}
& A=\left(a^{2}+c^{2}\right)\left(c^{2}+d^{2}\right)-(a b+c d)(a c+b d) \\
& B=\left(c^{2}+d^{2}\right)(a b+c d)-\left(b^{2}+d^{2}\right)(a c+b d) \\
& C=\left(a^{2}+b^{2}\right)(a b+c d)-\left(a^{2}+c^{2}\right)(a c+b d) \\
& D=\left(a^{2}+b^{2}\right)\left(b^{2}+d^{2}\right)-(a b+c d)(a c+b d)
\end{aligned}
$$

Let $\Delta=(a-d)^{2}+(b+c)^{2}$. From above we have

$$
\begin{aligned}
& A-D=-(b+c)(b-c)\left(a^{2}+b^{2}+c^{2}+d^{2}\right) \\
& B+C=(a-d)(b-c)\left(a^{2}+b^{2}+c^{2}+d^{2}\right) \\
& B-C=-(a+d)(b-c) \Delta
\end{aligned}
$$

and

$$
\begin{aligned}
A+D & =(b-c)^{2} \Delta+2(a d-b c)^{2} \\
& =(b-c)^{2} \Delta+2 .
\end{aligned}
$$

Now we observe that

$$
a^{2}+b^{2}+c^{2}+d^{2}=(a-d)^{2}+(b+c)^{2}+2(a d-b c)=\Delta+2,
$$

and thus we find that

$$
(A-D)^{2}+(B+C)^{2}=(b-c)^{2}(\Delta+2)^{2}\left((b+c)^{2}+(a-d)^{2}\right)=(b-c)^{2}(\Delta+2)^{2} \Delta .
$$

TheOrem 2.1. Let char $F=2$. If $|F|>2$ then $\mathrm{SL}_{2}(F)$ has a non-empty subset $X$ such that $\theta_{u}(X) \subseteq X$ and such that $1 \notin X$.

Proof. Let

$$
w=\left(\begin{array}{ll}
a & b \\
c & d
\end{array}\right) \in \mathrm{SL}_{2}(F) \text { and } \theta_{u}(w)=\left(\begin{array}{cc}
A & B \\
C & D
\end{array}\right)
$$


¿From above we have

$$
\begin{aligned}
A+D & =(b+c)^{2}(a+b+c+d)^{2} \\
B+C & =(a+d)(b+c)(a+b+c+d)^{2} \\
A+B+C+D & =(b+c)(a+b+c+d)^{3} .
\end{aligned}
$$

Thus $0 \in\{a+d, b+c, a+b+c+d\}$ if and only if $0 \in\{A+D, B+C, A+B+C+D\}$. Define $X$ to be the set of matrices $w$ such that $0 \notin\{a+d, b+c, a+b+c+d\}$. It follows that $\theta_{u}(X) \subseteq X$ and clearly the identity matrix is not in $X$. For $\mu \notin\{0,1\}$ we have

$$
t=\left(\begin{array}{cc}
1 & 1 \\
\mu & \mu+1
\end{array}\right) \in X
$$

The result follows.

THEOREM 2.2. Let char $F \neq 2$. If $|F|>3$ then $\mathrm{SL}_{2}(F)$ has a non-empty subset $X$ such that $\theta_{u}(X) \subseteq X$ and such that $\pm 1 \notin X$.

Proof. Write $F^{*}$ for the multiplicative group of $F$, define $Q=\left\{\mu^{2} \mid \mu \in F^{*}\right\}$ and let $N=F^{*} \backslash Q$. If $F=\mathbb{Q}$ or $F$ is finite then we have $Q \neq F^{*}$. However if $Q=F^{*}$ then $F$ clearly contains either $\mathbb{Q}$ or a field of order $p^{2}$ for some prime $p>2$; thus $\mathrm{SL}_{2}(F)$ contains either or $\mathrm{SL}_{2}(\mathbb{Q})$ or a group $\mathrm{SL}_{2}\left(p^{2}\right)$. Therefore in the proof below we may assume that $Q \neq F^{*}$.

We use the same notation as above for the entries of a matrix $w \in \mathrm{SL}_{2}(F)$ and of its image $\theta_{u}(w)$, and we write $\Delta=(a-d)^{2}+(b+c)^{2}$. Let $X$ be the set of matrices $w$ such that $a+d \neq 0, b-c \neq 0$ and $-2 \Delta \in N$. We proceed to show that $\theta_{u}(X) \subseteq X$.

Suppose that $w \in X$. Since $a+d, b-c$ and $\Delta$ are all non-zero, $B-C$ is also non-zero. Moreover $(b-c)^{2} \cdot(-2 \Delta) \in N$ so that $(b-c)^{2} \cdot(-2 \Delta) \neq 4$ and $A+D$ is non-zero. Now note that if $\Delta+2=0$, then $-2 \Delta=4 \in Q$, a contradiction. So $\Delta+2 \neq 0$ and hence

$$
-2\left((A-D)^{2}+(B+C)^{2}\right)=(b-c)^{2}(\Delta+2)^{2}(-2 \Delta) \in N .
$$

Thus we have now shown that $\theta_{u}(X) \subseteq X$. Clearly $\pm 1 \notin X$.

To complete the proof, we must show that $X$ is non-empty whenever $|F|>3$. If $-2 \in N$ then we have

$$
\left(\begin{array}{ll}
1 & 0 \\
1 & 1
\end{array}\right) \in X
$$

In particular, if $|F|=5$ then $-2 \in N$ and $X \neq \emptyset$. Suppose then that $-2 \in Q$, so that $|F| \geqslant 7$. We shall consider

$$
w=\left(\begin{array}{cc}
\mu & 0 \\
\lambda\left(\mu-\mu^{-1}\right) & \mu^{-1}
\end{array}\right),
$$


where $\mu^{2} \notin\{0, \pm 1\}$ and $\lambda \neq 0$. Since $|F| \geqslant 7$ there is an element $\mu$ with the required properties. The conditions on $\mu$ and $\lambda$ guarantee that both $a+d=\mu+\mu^{-1}$ and $b-c=$ $-\lambda\left(\mu-\mu^{-1}\right)$ are non-zero. We then find that

$$
-2 .\left((a-d)^{2}+(b+c)^{2}\right)=-2\left(\lambda^{2}+1\right)\left(\mu-\mu^{-1}\right)^{2},
$$

and so the requirement (for $w \in X$ ) that this be in $N$ is equivalent to $-2\left(\lambda^{2}+1\right) \in N$, or to $\lambda^{2}+1 \in N$, since $-2 \in Q$. Suppose that there is no $\lambda$ with this property; then for all $\lambda \in F^{*}$ we have $\lambda^{2}+1 \in Q \cup\{0\}$, and so for all $\lambda_{1}, \lambda_{2} \in F$ we have $\lambda_{1}^{2}+\lambda_{2}^{2} \in Q \cup\{0\}$. This implies that $-1=(-2)+1 \in Q$, so that there is an element $i$ with $i^{2}=-1$, and that for each $x \in F^{*}$ we have

$$
x=\frac{1}{4}\left((x+1)^{2}-(x-1)^{2}\right)=\frac{1}{4}\left((x+1)^{2}+((x-1) i)^{2}\right) \in Q .
$$

This is a contradiction to the assumption that $Q \neq F^{*}$ and the result follows.

\section{The case $G \cong \mathrm{Sz}(q)$}

We begin by recalling the definition of the Suzuki groups and explaining our notation for some of its elements. Let $m$ be a positive integer, write $q=2^{2 m+1}$ and $s=\sqrt{2 q}=2^{m+1}$, and let $F=\mathbb{F}_{q}$. For $\lambda \in F$, the maps $\lambda \mapsto \lambda^{s}$ and $\lambda \mapsto \lambda^{2}$ are automorphisms of $F$. Moreover, we have $\lambda^{s^{2}}=\lambda^{2}$ for all $\lambda \in F$. For $a, b \in F$, we define

$$
T(a, b):=\left(\begin{array}{cccc}
1 & 0 & 0 & 0 \\
a & 1 & 0 & 0 \\
a^{1+s}+b & a^{s} & 1 & 0 \\
a^{2+s}+a b+b^{s} & b & a & 1
\end{array}\right) .
$$

The set $H$ of elements $T(a, b)$ is a group of order $q^{2}$ with multiplication given by

$$
T(a, b) T(c, d)=T\left(a+c, a c^{s}+b+d\right) .
$$

For $k \in F^{*}$ we define

$$
D(k):=\left(\begin{array}{cccc}
k^{s / 2+1} & 0 & 0 & 0 \\
0 & k^{s / 2} & 0 & 0 \\
0 & 0 & k^{-s / 2} & 0 \\
0 & 0 & 0 & k^{-s / 2-1}
\end{array}\right) .
$$

The set $D$ of elements $D(k)$ is a group of order $q-1$ and we have $D(k)^{-1} T(a, b) D(k)=$ $T(a, b)^{D(k)}=T\left(a k, b k^{1+s}\right)$, so that the group $L$ generated by $H, D$ is the split extension $H \rtimes D$ and has order $q^{2}(q-1)$. Finally, we define

$$
z:=\left(\begin{array}{cccc}
0 & 0 & 0 & 1 \\
0 & 0 & 1 & 0 \\
0 & 1 & 0 & 0 \\
1 & 0 & 0 & 0
\end{array}\right)
$$


Clearly $\langle D, z\rangle$ is a dihedral group. Let $G=\langle L, z\rangle$. Then each element of $G \backslash L$ can be written uniquely in the form $h_{1} d z h_{2}$ with $h_{1}, h_{2} \in H, d \in D$, and so $|G|=q^{2}(q-1)\left(q^{2}+1\right)$. The group $G$ is isomorphic to the Suzuki group $\mathrm{Sz}(q)$ : this is the description given by Suzuki [?, p. 133], but with slightly different notation.

THEOREM 3.1. For all $q \geqslant 8$ the group $\mathrm{Sz}(q)$ has an involution $u$ and a non-empty subset $X$ with $\theta_{u}(X) \subseteq X$ and $1 \notin X$.

Proof. We begin by defining the element $u$ and the set $X$. Let

$$
u=T(0,1)=\left(\begin{array}{llll}
1 & 0 & 0 & 0 \\
0 & 1 & 0 & 0 \\
1 & 0 & 1 & 0 \\
1 & 1 & 0 & 1
\end{array}\right)
$$

Then $u$ is an involution in $H$ and $\mathrm{C}_{G}(u)=H$. Now define $Y=Y_{1} \cup Y_{2} \cup Y_{3}$ and $X=G \backslash Y$ where

(i) $Y_{1}=L$,

(ii) $Y_{2}$ consists of the matrices in $G$ of trace 0 , and

(iii) $Y_{3}$ consists of those $g \in G$ such that $(u g)^{2}=1$.

Clearly $1 \notin X$. We also observe immediately that $X$ is non-empty. Indeed, choose $b \notin\{0,1\}$, and consider the matrix

$$
x:=T(0, b) D(1) z=T(0, b) z=\left(\begin{array}{cccc}
0 & 0 & 0 & 1 \\
0 & 0 & 1 & 0 \\
0 & 1 & 0 & b \\
1 & 0 & b & b^{s}
\end{array}\right) .
$$

Thus $x \notin Y_{1}=L$, and $x$ has trace $b^{s}$, so that $x \notin Y_{2}$. Finally, we calculate that the $(1,3)$-entry of $(u x)^{2}$ is $b+1 \neq 0$, so that $x \notin Y_{3}$. Therefore $x \in X$.

We shall prove that $\theta_{u}(X) \subseteq X$ by showing that

$$
\text { if } \theta_{u}(w) \in Y \text { then } w \in Y \text {. }
$$

We begin by making a reduction, using the fact that each of the sets $Y_{1}, Y_{2}$ and $Y_{3}$ is invariant under conjugation by the elements of $H=\mathrm{C}_{G}(u)$. Clearly we may restrict attention to elements $w$ not in $Y_{1}=L$; each such element $w$ is in $H D z H$ and so can be written in the form $\tilde{w}^{h}$ with $\tilde{w} \in H D z$. The invariance of each $Y_{i}$ under $H$ and the fact that $\theta_{u}\left(\tilde{w}^{h}\right)=\left(\theta_{u}(\tilde{w})\right)^{h}$ (since $h \in H=\mathrm{C}_{G}(u)$ ) show that $w \in Y$ if and only if $\tilde{w} \in Y$ and that $\theta_{u}(w) \in Y_{2} \cup Y_{3}$ if and only if $\theta_{u}(\tilde{w}) \in Y_{2} \cup Y_{3}$. Therefore it is sufficient to prove that

$$
\text { if } \tilde{w} \in H D z \text { and } \theta_{u}(\tilde{w}) \in Y \text { then } \tilde{w} \in Y_{2} \cup Y_{3} \text {. }
$$

Let $\tilde{w}$ be an element of $H D z$ such that $\theta_{u}(\tilde{w}) \in Y$. Write $\tilde{w}=T(a, b) D(k) z$ where $a, b, k \in F$ and $k \neq 0$, and write $\theta_{u}(\tilde{w})=M=\left(M_{i j}\right)$. 
Lemma 3.2. The entries $M_{i j}$ of the matrix $M$ are as follows:

$$
\begin{aligned}
& M_{11}=1+k^{s+1}(b+1)+k^{s+2} \xi \\
& M_{12}=k^{s+1} a^{s}+k^{s+2}\left(a^{s+1}+b+1\right) \\
& M_{13}=k^{s+2} a+k^{2 s+2}(b+1)+k^{2 s+3} \xi \\
& M_{14}=k^{2 s+2} a^{s}+k^{2 s+3} a^{s+1}+k^{2 s+4} \xi \\
& M_{21}=k^{s+1}\left(a^{s+2}+b^{s}+1\right)+k^{s+2} a \xi \\
& M_{22}=1+k^{s+1}(b+1)+k^{s+2}\left(a^{s+2}+a b+a\right) \\
& M_{23}=k^{s+2} a^{2}+k^{2 s+2}\left(a^{s+2}+b^{s}+1\right)+k^{2 s+3} a \xi \\
& M_{24}=k^{s+2} a+k^{2 s+2}(b+1)+k^{2 s+3}\left(a^{s+2}+\xi\right)+k^{2 s+4} a \xi \\
& M_{31}=M_{42}=k^{s+1} \mu+k^{s+2} \nu \\
& M_{32}=k^{s+2}\left(a^{s+1}+b+1\right)^{2} \\
& M_{33}=1+k^{s+1}(b+1)+k^{s+2}\left(a^{s+2}+a b+a\right)+k^{2 s+2} \mu+k^{2 s+3} \nu \\
& M_{34}=k^{s+1} a^{s}+k^{s+2}\left(a^{s+1}+b+1\right)+k^{2 s+3}\left(a^{s} b^{s}+a^{s}\right)+k^{2 s+4} \nu \\
& M_{41}=k^{s+2} \xi^{2} \\
& M_{43}=k^{s+1}\left(a^{s+2}+b^{s}+1\right)+k^{s+2} a \xi+k^{2 s+3} \xi^{2} \\
& M_{44}=1+k^{s+1}(b+1)+k^{s+2} \xi+k^{2 s+2} \mu+k^{2 s+3} \nu+k^{2 s+4} \xi^{2},
\end{aligned}
$$

where

$$
\begin{aligned}
& \mu=a^{2 s+2}+a^{s} b^{s}+a^{s}+b^{2}+1 \\
& \nu=a \mu+(b+1)\left(b^{s}+1\right) \\
& \xi=a^{s+2}+b^{s}+a b+a+1 .
\end{aligned}
$$

The proof of the lemma is a routine but tedious calculation which can be carried out either by hand or using one of the standard computer algebra packages such as MAGMA $[?]$.

We proceed now to prove the assertion $(*)$.

Case 1. Suppose that $M \in Y_{1}=L$.

Here we have $M_{12}=M_{13}=M_{14}=0$, and hence

$$
k^{s+1} M_{12}+k M_{13}+M_{14}=a k^{s+3}=0 .
$$

Thus $a=0$ since $k \neq 0$. The equation $M_{12}=0$ now becomes $k^{s+2}(b+1)=0$, so that $b=1$. Therefore $\tilde{w}=T(0,1) D(k) z$ for some $k \in F^{*}$; but then $u \tilde{w}=D(k) z$ is an involution in the dihedral group $D\langle z\rangle$, and thus $\tilde{w} \in Y_{3}$.

Case 2. Suppose that $M \in Y_{2}$.

Here we have $M_{11}+M_{22}+M_{33}+M_{44}=0$. This gives the equation $k^{2 s+4} \xi^{2}=0$, and so since $k \neq 0$ we have $\xi=0$. It follows that

$$
a^{s} \xi+\xi^{s}=a^{s+1} b+a^{s+1}+b^{2}+1=0,
$$


giving $a^{s+1}(b+1)+(b+1)^{2}=0$, whence $b=1$ or $a^{s+1}=b+1$. If $a^{s+1}=b+1$ then the equation $\xi=0$ becomes $b^{s}+1=0$, and we obtain that $b=1$.

Therefore $b=1$. Now the equation $\xi=0$ becomes $a^{s+2}=0$ and we have $a=0$. Thus $\tilde{w}=T(0,1) D(k) z$, and we have already seen that this element lies in $Y_{3}$.

Case 3. Suppose that $M \in Y_{3}$.

We need to determine the form of the typical element of $Y_{3} \backslash Y_{1}$. We recall that $Y_{3}, Y_{1}$ are invariant under conjugation by $H=\mathrm{C}_{G}(u)$. Each element $g$ of $G \backslash Y_{1}$ has the form $\tilde{g}^{T(c, d)}$ where $\tilde{g} \in H D z \subseteq G \backslash Y_{1}$, and $g \in Y_{3}$ if and only if $\tilde{g} \in Y_{3}$. Writing $\tilde{g}=T(\alpha, \beta) D(\lambda) z$, we have $(u \tilde{g})^{2}=1$ if and only if $(T(\alpha, \beta+1) D(\lambda) z)^{2}=1$, or equivalently if and only if

$$
T(\alpha, \beta+1) D(\lambda)=z D(\lambda)^{-1}\left(T(\alpha, \beta+1)^{-1}\right) z
$$

Since the right-hand side here is an upper-triangular matrix this equation implies that $\alpha=0$ and $\beta=1$. Thus the typical element of $Y_{3} \backslash Y_{1}$ has the form

$$
N:=(T(0,1) D(l) z)^{T(c, d)}
$$

where $c, d, l \in F$ and $l \neq 0$.

Therefore, we must solve the equation $M=N$. Another calculation gives some of the matrix entries of $N$ :

Lemma 3.3. Some entries $N_{i j}$ of the matrix $N=(T(0,1) D(l) z)^{T(c, d)}$ are as follows:

$$
\begin{aligned}
& N_{11}=l^{s / 2+1}\left(c^{s+2}+d^{s}+c d\right) \\
& N_{12}=l^{s / 2+1} d \\
& N_{13}=N_{24}=l^{s / 2+1} c \\
& N_{14}=l^{s / 2+1} \\
& N_{22}=l^{s / 2} c^{s}+l^{s / 2+1} c d \\
& N_{31}=l^{-s / 2} c+l^{s / 2}\left(c^{2 s+1}+c^{s} d\right)+l^{s / 2+1}\left(c^{s+2}+c d+d^{s}+c^{s+2} d+c d^{2}+d^{s+1}\right) \\
& N_{34}=l^{s / 2+1}(d+1) \\
& N_{42}=l^{-s / 2} c+l^{s / 2}\left(c^{2 s+1}+c^{s} d+c^{s}\right)+l^{s / 2+1}\left(c^{s+2} d+d^{s+1}+c d^{2}+c d+d\right) .
\end{aligned}
$$

¿From the information in this lemma we have (for all values of $c, d$ and $l$ )

$$
\begin{gathered}
N_{11}+N_{12}+N_{22}+N_{31}+N_{42}=0, \\
N_{13}+N_{24}=0 \\
N_{12}+N_{14}+N_{34}=0 .
\end{gathered}
$$

Considering the three corresponding equations for entries of $M$, and dividing by $k^{s+2}$, $k^{2 s+4}, k^{2 s+4}$, we obtain the equations

$$
a^{s+1}+b+a^{s} k^{-1}+b^{s}=0,
$$




$$
\begin{gathered}
a^{s+3}+a^{2} b+a^{s+2} k^{-1}+a^{2}+a b^{s}+a=0, \\
a^{2 s+3}+a^{s+2}+a b^{2}+a b+a^{s+1} b^{s}+a^{s+1}+b^{s+1}+b+\left(a^{s+1}+a^{s} b^{s}+a^{s}\right) k^{-1}+a^{s} k^{-2}=0 .
\end{gathered}
$$

Multiplying Equation (1) by $a^{2}$ and adding (2) we obtain

$$
a^{2} b^{s}+a^{2}+a b^{s}+a=a(a+1)\left(b^{s}+1\right)=0,
$$

so that either $a \in\{0,1\}$ or $b=b^{s^{2}} b^{-1}=b^{-1}$ and hence $b=1$.

First suppose that $a=0$. Then (1) becomes $b+b^{s}=0$, and so we have $b=b^{s}=b^{s^{2}}=b^{2}$ and hence $b=0$ or $b=1$. If $(a, b)=(0,0)$ then $\tilde{w}=D(k) z$, which has trace 0 , and so lies in $Y_{2}$. The case $(a, b)=(0,1)$ has arisen before: we have $T(0,1) D(k) z \in Y_{3}$.

Next suppose that $b=1$ and $a \neq 0$. Then (1) gives $a^{s}\left(a+k^{-1}\right)=0$ and so we have $a=k^{-1}$. Equation (3) yields that $a^{s+1}+1=0$ and hence $a=a^{s^{2}-1}=\left(a^{s+1}\right)^{s-1}=1$.

Suppose that $a=1$. We note that for all $\beta \in F, \kappa \in F^{*}$ the matrix $T(1, \beta) D(\kappa) z$ has diagonal entries $0,0, \kappa^{s / 2}, \kappa^{s / 2+1}\left(\beta+\beta^{s}+1\right)$ and hence trace $\kappa^{s / 2}\left(1+\kappa\left(\beta+\beta^{s}+1\right)\right)$. Thus when $a=1$, Equation (1) yields that $\left(b+b^{s}+1\right)+k^{-1}=0$ and that $\tilde{w}=T(1, b) D(k) z$ has trace 0 and lies in $Y_{2}$.

This concludes the proof of Theorem 3.1.

\section{References}

[1] T. Bandman, G.-M. Greuel, F. Grunewald, B. Kunyavskiı, G. Pfister and E. Plotkin. Engel-like identities characterizing finite soluble groups. arXiv:math.GR/0303165 v1, 13 March 2003.

[2] Rolf Brandl and John S. Wilson. Characterization of finite soluble groups by laws in a small number of variables. J. Algebra 116 (1988), 334-341.

[3] J. J. Cannon et al. The Magma programming language, version 2.8. School of Mathematics and Statistics, University of Sydney (2001).

[4] F. Grunewald, B. Kunyavskiǔ, D. Nikolova and E. Plotkin. Two-variable identities in groups and Lie algebras. Zap. Nauchn. Sem. S.-Peterburg Otdel. Mat. Inst. Steklov. (POMI) 272 (2000), 161-176.

[5] D. J. S. Robinson. A course in the theory of groups (Springer-Verlag, 1982).

[6] M. Suzuki. On a class of doubly transitive groups. Ann. of Math. (2) 75 (1962), $105-145$.

[7] J. G. Thompson. Nonsolvable groups all of whose local subgroups are solvable. Bull. Amer. Math. Soc. 74 (1968), 383-437. 
Authors' addresses

John N. Bray, School of Mathematics and Statistics, University of Birmingham, Edgbaston, Birmingham B15 2TT

E-mail: jnb@maths.bham.ac.uk

John S. Wilson, Mathematical Institute, 29-29 St Giles', Oxford OX1 3LB

E-mail: wilsonjs@maths.ox.ac.uk

Robert A. Wilson, School of Mathematics and Statistics, University of Birmingham, Edgbaston, Birmingham B15 2TT

E-mail: raw@maths.bham.ac.uk 